\title{
A Utilização de Softwares no Ensino de Matemática para Ensino Fundamental e Médio
}

\author{
Paul Symon Ribeiro Rocha1, Carlos Vieira Ramos2, Tainara Antunes Brasil3 \\ 1Instituto Federal de Educação, Ciência e Tecnologia do Maranhão - IFMA | Campus \\ Barreirinhas
}

2Instituto Federal de Educação, Ciência e Tecnologia do Piauí | Universidade Aberta Do Brasil - UAB / IFPI

3Universidade Federal do Piauí - UFPI

paul.rocha@ifma.edu.br, \{carlosvieiraramos1, tainaraantunesbrasil\}@gmail . com

\begin{abstract}
The present work sought to explore the importance of technology for teaching and learning in the area of mathematics. It is indicated the use of software as a methodological resource. It presents a mapping on math software for the aid of elementary and middle school teachers. The general objective is to analyze how educational software can contribute to and assist in the process of teaching and learning the content programmed by math teachers. It is hoped that this study will contribute with teachers in the expansion of the theory of the use of technology, as a way to provide an alternative resource to arouse the interest of the students as well as to assist in the learning of Mathematics.
\end{abstract}

Resumo. O presente trabalho buscou explorar a importância da tecnologia para o ensino e aprendizagem na área de matemática. Indica-se a utilização de softwares como um recurso metodológico. Apresenta um mapeamento sobre os softwares de matemática para o auxílio de professores de ensino fundamental e médio. O objetivo geral é analisar como os softwares educacionais podem contribuir e auxiliar no processo de ensino e aprendizagem do conteúdo programado pelos professores de matemática. Espera-se com este estudo contribuir com os professores na ampliação da teoria do uso da tecnologia, como forma de proporcionar recurso alternativo para despertar o interesse dos estudantes além de auxiliar na aprendizagem de Matemática.

\section{Introdução}

A Constituição Federal do Brasil, de 1988, Art. 205, dentro do rol dos direitos humanos fundamentais encontra-se o direito à educação, amparado por normas nacionais e internacionais. Trata-se de um direito fundamental, porque inclui um processo de desenvolvimento individual próprio à condição humana. 
Além dessa perspectiva individual, esse direito deve ser visto, sobretudo de forma coletiva, como um direito a uma política educacional, a ações afirmativas do Estado que oferecem à sociedade instrumentos para alcançar seus fins.

\begin{abstract}
Art. 205. A educação, direito de todos e dever do Estado e da família, será promovida e incentivada com a colaboração da sociedade, visando ao pleno desenvolvimento da pessoa, seu preparo para o exercício da cidadania e sua qualificação para o trabalho. [Brasil 1988]
\end{abstract}

A matemática nas escolas ainda é vista como algo fragmentado, com ensinos mecanizados, descontextualizados, com base em memorização, levantando uma barreira quanto ao real objetivo do ensino, proporcionar situações concretas de como essa disciplina pode ser utilizada no cotidiano. Diante dessa colocação, os Parâmetros Curriculares Nacionais (PCN) reforçam:

[...]o ensino de Matemática prestará sua contribuição à medida que forem exploradas metodologias que priorizem a criação de estratégias, a comprovação, a justificativa, a argumentação, o espírito crítico, e favoreçam a criatividade, o trabalho coletivo, a iniciativa pessoal e a autonomia advinda do desenvolvimento da confiança na própria capacidade de conhecer e enfrentar desafios. [BRASIL 1997]

[Machado 1994], seguindo a mesma linha de pensamento, reforça ainda mais essa ideia, quando afirma que a escola deve expandir ainda mais as fronteiras das disciplinas, buscando por meio do computador, em especial os jogos computadorizados, contribuir com este processo, abrindo um leque de oportunidades para interagir com outros saberes, como a matemática, geografia e a história, envolvendo mapas, utilizando escalas, transformações de unidades de medidas e tantos outros temas de forma criativa, envolvendo a música, arte, trabalhando de maneira transdisciplinar.

\title{
2. Referencial teórico
}

Nesta seção, é apresentado um breve referencial teórico sobre as áreas envolvidas neste trabalho, destacando conceitos sobre o uso do computador como metodologia de ensino e softwares educacionais.

\subsection{O uso do computador como metodologia de ensino}

A inserção da Informática aplicada à Educação tem sido um fator preponderante no âmbito educacional, proporcionando aos professores ampliar os métodos de ensino e, aos alunos, ampliar as chances de aprendizagem.

[Silva et al. 2013] afirma como o uso de computadores no processo de ensino aprendizagem possibilita, não só ao aluno, mas ao professor também, pois torna um ensino mais dinâmico. Porém a escola deve adotar um plano pedagógico para a disciplina especifica, aplicando o uso do computador como uma ferramenta útil e viável e não um impasse ao ensino.

A utilização do computador como aliado aos processos de ensino e aprendizagem tornou-se essencial na vida de todos. De acordo com [Tederke, Fortes e Silveira 2016], as crianças desde pequenas já possuem uma habilidade incrível no manuseio das tecnologias, e a partir daí, as propostas escolares podem incluir estas tecnologias nas salas de aula, em forma de jogos educacionais e pelo emprego de softwares, englobando as diversas áreas do conhecimento.

Ainda na mesma linha de pensamento, [Tederke, Fortes e Silveira 2016], a aplicação da Informática como apoio aos processos de ensino e de aprendizagem pode 
ser realizada por meio da utilização de diversos softwares, tais como editores de texto, jogos educacionais digitais, simuladores, entre outros. Qualquer software que seja utilizado como apoio aos processos de ensino e de aprendizagem pode ser considerado um software educacional.

\subsection{Softwares Educacionais}

Os Softwares Educacionais foram criados com o objetivo de auxiliar nos processos de ensino e aprendizagem, fazendo com que os educandos iniciem ou aprimorem seus conhecimentos, tanto de informática, como das áreas do conhecimento em que os softwares forem inseridos. O que diferencia um software educacional dos demais é o fato dele ter sido desenvolvido com o propósito de ensino e aprendizagem e não apenas para diversão [Morais 2003].

Para o uso dos recursos de informática, é necessário que os professores possuam uma boa habilidade com o uso dos softwares escolhidos para apresentar aos alunos. Os Parâmetros Curriculares Nacionais (PCN) de Matemática, publicados em 1997, já orientavam que os docentes devem estar familiarizados com o material pedagógico a ser utilizado em sala de aula, pois assim os educadores conseguirão integrar o uso da tecnologia com o ensino de Matemática, fazendo como que essa integração torne o aprender dos alunos algo eficiente [Brasil 1997].

\section{Metodologia}

A pesquisa realizada configura-se como uma Revisão Sistemática, que de acordo com [Cordeiro et al. 2007], consiste em um tipo de investigação que objetiva "reunir, avaliar criticamente e conduzir uma síntese dos resultados de múltiplos estudos primários".

\subsection{Objetivo de Pesquisa}

Esta Revisão Sistemática tem como objetivo identificar e analisar os softwares de ensino de matemática para o auxílio de professores de ensino fundamental e médio.

\subsection{Questão de Pesquisa}

Foi definida uma questão primária principal, para esta Revisão Sistemática:

- Questão primária (QP): Quais os principais softwares desenvolvidos para auxiliar os professores de matemática, no ensino fundamental e médio?

\subsection{Critérios de Inclusão e Exclusão para Seleção de Estudos}

- Critérios de Inclusão: Artigos, Monografias e Dissertações que abordam softwares para o ensino de matemática no ensino fundamental e médio a partir do ano de 2004.

- Critérios de Exclusão: Artigos, monografias e dissertações que não abordam softwares para o ensino de matemática no ensino fundamental e médio, ou, que foram publicados antes de 2004.

\subsection{Processo de Seleção dos Estudos}

Utilizou-se, como fonte de dados, a literatura sobre o uso Softwares no ensino de matemática, a partir de buscas nos portais: REMAT (Revista Eletrônica da Matemática): https://periodicos.ifrs.edu.br/index.php/REMAT/search, Secretaria da Educação: http://www.educacao.pr.gov.br/, Bienal - Sociedade Brasileira de Matemática http://www.mat.ufpb.br/bienalsbm/, CONEDU - CONGRESSO NACIONAL DE EDUCAÇÃO http://www.conedu.com.br/2016/, REVISTA DIALOGOS http://www.revistadialogos.com.br/, TISE (Congresso Internacional de Informática 
Educativa) http://www.tise.cl/ e o Google Acadêmico: https://scholar.google.com.br, usando as palavras-chaves "matemática", "softwares" e "ensino". Feita a busca, retornaram 11 trabalhos que abordam a temática pesquisada.

Após o mapeamento das produções científicas, realizou-se um estudo exploratório a partir da leitura dos resumos dos artigos, monografias e dissertações para análise geral do tema proposto e seleção dos que abordassem a temática de interesse do estudo, para a partir de então, iniciar a análise mais aprofundada dos trabalhos selecionados.

\section{Condução da Revisão}

Dos 11 (onze) trabalhos encontrados, 8 (oito) são artigos, 1 (uma) monografia, 2 (duas) dissertações. Os trabalhos selecionados para análise são apresentados na Tabela 1 (artigos) e na Tabela 2 (monografia e dissertações).

Tabela 1 - Artigos com a temática Softwares no ensino de Matemática

\begin{tabular}{|c|c|c|c|}
\hline SOFTWARE & AUTOR/ANO & REVISTA & ANO/SERIE \\
\hline GEOGEBRA & [Fanti 2010] & $\begin{array}{c}\text { V Bienal } \\
\text { SBM/UFPB }\end{array}$ & $\begin{array}{c}\text { Ensino } \\
\text { Fundamental e } \\
\text { Médio }\end{array}$ \\
\hline $\begin{array}{c}\text { Trigonométricos, } \\
\text { Geométricos, } \\
\text { Gráficos, } \\
\text { Recreativos, } \\
\text { Algébricos, Notação } \\
\text { Matemática, } \\
\text { Estatísticos e } \\
\text { Multidisciplinares. }\end{array}$ & $\begin{array}{l}\text { [Contri, Retzlaff e Klee } \\
\text { 2011] }\end{array}$ & CNEM & $\begin{array}{c}\text { Ensino } \\
\text { Fundamental e } \\
\text { Médio }\end{array}$ \\
\hline SUPERLOGO & [Nogueira et al. 2013] & TISE & $\begin{array}{l}\text { Ens. Fundamental } \\
6^{\circ} \text { ao } 9^{\circ}\end{array}$ \\
\hline $\begin{array}{l}\text { Aplicativos e } \\
\text { Educativos }\end{array}$ & $\begin{array}{l}\text { [Pacheco e Barros } \\
\text { 2013] }\end{array}$ & DIÁLOGOS & $\begin{array}{l}\text { Ens. Fundamental e } \\
\text { Médio }\end{array}$ \\
\hline GEOGEBRA & [Pereira 2014] & $\begin{array}{c}\text { Sec. de } \\
\text { Educação PR } \\
\end{array}$ & Ensino Médio \\
\hline GEOGEBRA & $\begin{array}{l}\text { [Tederke, Fortes e } \\
\text { Silveira 2016] }\end{array}$ & REMAT & $\begin{array}{c}3^{\circ} \text { ano Ensino } \\
\text { Médio }\end{array}$ \\
\hline $\begin{array}{c}\text { MATH } \\
\text { CHALLENGE } \\
\text { FREE. (Aritmética } \\
\text { Mental) }\end{array}$ & [Costa et al. 2016] & III CONEDU & $\begin{array}{l}5^{\circ} \text { ano Ens. } \\
\text { Fundamental }\end{array}$ \\
\hline GEOGEBRA & $\begin{array}{l}\text { [Silva, Ribeiro e Araújo } \\
\text { 2018] }\end{array}$ & CONEDU & Ensino Médio \\
\hline
\end{tabular}

Dos artigos selecionados, o estudo "Utilizando o software GeoGebra no Ensino de certos conteúdos matemáticos", [Fanti 2010], descreve como objetivo do trabalho introduzir as noções básicas do programa GeoGebra e utilizá-lo no estudo de certos conteúdos matemáticos como polígonos, funções reais, e em especial no estudo das 
cônicas. São inúmeras as aplicações das cônicas no cotidiano. [Fanti 2010], explica as principais atividades realizadas pelo software.

O artigo "Uso de softwares matemáticos como facilitador da aprendizagem", que tem como autores [Contri, Retzlaff e Klee 2011] tem por objetivo principal a pesquisa aprimorar o conhecimento dos acadêmicos através da utilização e aplicação de softwares estatísticos e matemáticos, objetivando uma maior interação entre conteúdos, para o aprimoramento das práticas pedagógicas. $\mathrm{O}$ trabalho buscou promover $\mathrm{o}$ desenvolvimento científico e tecnológico na área de conhecimento da matemática, por meio de recursos tecnológicos. A pesquisa foi desenvolvida na Universidade Regional Integrada do Alto Uruguai e das Missões-URI, que possui diversos cursos de graduação. Os softwares mencionados na pesquisa foram: trigonométricos - círculo trigonométricos e thales; geométricos - geogebra, régua e compasso e wingeom; gráficos - graphmática e winplot; recreativos - torre de hanói e winarc; algébricos -determinante, winmat e winmatrix; notação matemática - math type; estatísticos - biostat e estatistica; e multidisciplinares - free mat, matlab e mupad.

O trabalho "Softwares educativos gratuitos para o ensino de Matemática", que tem como autor [Nogueira et al. 2013] tem como objetivo elaborar um catálogo com softwares educativos gratuitos para o ensino de Matemática do $6^{\circ}$ ao $9^{\circ}$ ano do ensino fundamental, assim como classificá-los pela forma que o aluno interage com $o$ computador. O mapeamento conta com inúmeros softwares que podem ser utilizados para conteúdo de Matemática, divididos em modalidades. Os softwares encontrados foram: A magia dos Números, Círculo 0, Shapari, Torre de Hanói, Torus Games (Kali), Trilha Matemática, TuxMath e Winarc. Porém, a pesquisa constatou que o único software gratuito é o SUPERLOGO, versão moderna e inspirada na linguagem.

Além disso, o autor dá ênfase as dificuldades encontradas pelos professores em utilizar esses softwares. Assim, as dificuldades começam desde a seleção dos softwares, o domínio técnico da máquina, a articulação entre os tópicos de conteúdo, a maneira como os professores trabalham didaticamente com os alunos e as possíveis intervenções a serem feitas durante a sua utilização.

Analisando o artigo "O Uso de Softwares Educativos no Ensino de Matemática", de [Pacheco e Barros 2013], essa pesquisa avalia a utilização de softwares educativos de matemática na sala de aula e suas contribuições ao processo de ensino aprendizagem.

A pesquisa intitulada "O Ensino e a Aprendizagem da Matemática mediada por softwares Educativo na forma de Objetos de Aprendizagem" tem como objetivo analisar a utilização de softwares educativos, na forma de Objetos de Aprendizagem (OA), nos processos de ensino e aprendizagem de conteúdos matemáticos, a fim de identificar qual a relevância do uso das tecnologias em forma de OA, na prática pedagógica de professores de matemática em sala de aula.

O GeoGebra foi escolhido como objeto de pesquisa, por ser um software gratuito de matemática dinâmica desenvolvido para o ensino e aprendizagem da matemática nos vários níveis de ensino. O GeoGebra reúne recursos de geometria, álgebra, tabelas, gráficos, probabilidade, estatística e cálculos simbólicos em um único ambiente. Permite a realização de diferentes atividades, entre elas, a construção de pontos, segmentos de reta, retas paralelas e perpendiculares, construção de gráficos de funções, construção de figuras geométricas, permite ainda calcular o ponto médio dos segmentos, a área, o perímetro das figuras, medir ângulos, entre outras. 
O artigo "Um Estudo de Caso Envolvendo a Aplicação de um Software Educacional de Geometria Espacial" tem por objetivo principal, realizar um estudo de caso voltado a alunos de $3^{\circ}$ ano do Ensino Médio, envolvendo a aplicação de um software educacional - o GeoGebra - no estudo de tópicos de Geometria Espacial. De acordo com [Tederke, Fortes e Silveira 2016] este trabalho visa proporcionar a inclusão de ferramentas tecnológicas nos processos de ensino e de aprendizagem, que poderão ser estendidas após a realização deste estudo de caso trazendo, assim, resultados positivos para alunos e professores.

Para [Tederke, Fortes e Silveira 2016] o software GeoGebra foi o escolhido para o estudo de caso justamente porque favorece a construção de figuras geométricas (planas e espaciais) e a movimentação dos elementos dessas figuras, alterando formatos e medidas de forma dinâmica. Assim, o aluno pode perceber as relações existentes entre os elementos dessas figuras e constatar propriedades, facilitando a assimilação dos conceitos e definições referentes a essas figuras geométricas.

$O$ artigo "O uso de softwares aplicativos no ensino da Matemática: a tecnologia como figura de mediação pedagógica" de [Costa et al. 2016] demonstra que trabalho em questão é síntese de uma pesquisa ainda em desenvolvimento sobre o uso de softwares na educação matemática, o enfoque central é o uso de um aplicativo que trabalha as quatro operações. "O MATH CHALLENGE FREE", o mesmo está disponível na playstore que é o ambientem de download nos dispositivos android. Partindo da aplicação de alguns testes, analisa-se sobre que dificuldades que os alunos possuem sobre operações básicas e então usamos o aplicativo como proposta de intervenção.

O objetivo em questão é mostrar como os aplicativos podem ser utilizados como artifício na construção do "fazer matemática", englobando na prática uma educação com sentido. Quando se fala no uso da tecnologia na educação a primeira coisa que se imagina é o computador, contudo, os softwares educacionais não se restringem apenas ao uso dele, os smartphones, tablets, dentre outros aparelhos de tecnologia móvel de comunicação podem ser inseridos desse contexto.

E por fim, o artigo "O uso de softwares no ensino da matemática: entre o modismo e o uso inteligente" dos autores [Silva, Ribeiro e Araújo 2018] investigou o uso de softwares educacionais voltados para o ensino-aprendizagem da matemática, utilizados de forma inteligente ou pelo modismo dos professores de matemática de três escolas públicas da rede estadual de ensino de Manaus/Amazonas.

[Silva, Ribeiro e Araújo 2018] afirmam que a pesquisa demonstrou que o software matemático Geogebra está em evidência, e tornou-se uma moda entre os professores de matemática devidos algumas características do mesmo: software gratuito, fácil manipulação, já possui toda sua estrutura e plataforma traduzida para a língua portuguesa, pode ser acessado pelo celular, seus recursos gráficos em 2 e $3 \mathrm{D}$, e podendo ser utilizado como ferramenta educacional para qualquer série do ensino básico estes fatos relatados pelos professores de matemática. A pesquisa deixou claro que alguns professores de matemática não sabiam a real vantagem educacional por traz de um software, e que a transposição didática que poderia ocorre quando utilizavam esta ferramenta educacional que é o software.

A Tabela 2 apresenta as monografias e dissertações encontradas e analisadas neste trabalho:

Tabela 2 - Monografia e dissertações encontradas a partir da busca realizada. 


\begin{tabular}{|c|c|c|c|}
\hline SOFTWARE & AUTOR/ANO & PROGRAMA & ANO/SERIE \\
\hline $\begin{array}{c}\text { EDUCACION } \\
\text { AL } \\
\text { (jogos) }\end{array}$ & [Abreu 2011] & $\begin{array}{c}\text { Pós-graduação } \\
\text { UFMT/UAB }\end{array}$ & $\begin{array}{c}\text { Ensino } \\
\text { Fundamental }\end{array}$ \\
\hline SOFTMAT & [Batista 2004] & $\begin{array}{c}\text { Dissertação } \\
\text { CCT/UENF }\end{array}$ & Ensino Médio \\
\hline $\begin{array}{c}\text { GEOBGEBRA } \\
\text { e Método ABP }\end{array}$ & [Silva 2015] & $\begin{array}{c}\text { Mestrado em Ensino de } \\
\text { Ciências e Matemática } \\
\text { UCS/SP }\end{array}$ & $\begin{array}{c}1^{\text {o }} \text { ano Ensino } \\
\text { Médio }\end{array}$ \\
\hline
\end{tabular}

A monografia intitulada "O uso de softwares na aprendizagem da matemática", o autor [Abreu 2011], trata das possibilidades de se trabalhar a matemática, com alunos que apresentam baixo rendimento em sala de aula na disciplina da matemática, enfocando os jogos educativos como um novo recurso na educação. Propõese a análise dos jogos educativos.

Foi selecionado alguns softwares para o estudo para a análise de pontos para o ensino de matemática, entre eles temos: Site Smart Kids, Software Sebran, Site Atividades Educativas, Tux Math, Ahsha Math, Tangran, Software Torre de Hanói.

[Abreu 2011] expõe resultados demonstraram melhoria na aprendizagem dos alunos em relação à aprendizagem da matemática, observado em todos os alunos. $\mathrm{O}$ emprego dos softwares educativos foi bem aceito pelo grupo, pois atraiam as crianças. As crianças antes de desenvolverem as atividades de matemática no computador eram desmotivadas com a disciplina de matemática, os alunos não utilizavam de jogos, não eram usuárias de computador e passaram a utilizar as ferramentas sem dificuldades apresentavam se mais atentas na sala. A mediação estabelecida pelo software educativo apresentou o despertar na busca do conhecimento. Analisa-se que para resolução de cálculos matemáticos as crianças apresentaram melhor conhecimentos dos conteúdos, os jogos melhoram o processo de aprendizagem das crianças na disciplina trabalhada.

[Batista 2004] apresenta sua dissertação com o título "Softmat: um repositório de softwares para matemática" objetiva incentivar posturas conscientes e críticas em relação à seleção de softwares educacionais, através da disponibilização do SoftMat, um repositório contendo um conjunto de softwares para Matemática do Ensino Médio, devidamente acompanhados de suas avaliações de qualidade do ensino médio - um instrumento em prol de posturas mais conscientes na seleção de softwares Educacionais.

A seguir, foi feito um levantamento de softwares selecionados. [Batista 2004] ressalva que os softwares que estão disponíveis para download no SoftMat, ou são livres ou são proprietários com autorização de seus responsáveis. Os principais softwares são: Régua e Compasso, SuperLogo, Winplot, Winmat, Ms. Lindquist, Poly, Venn, NCX Círculo Trigonométrico, Resolução de Sistemas Lineares, OpenOficce.org Calc.

Nesta dissertação a principal preocupação foi incentivar o uso de softwares educacionais de forma consciente e crítica. [Batista 2004] objetivou despertar potenciais usuários de softwares educacionais para a importância de avaliar a qualidade dos mesmos. Qualidade que deve estar presente não só nos softwares educacionais como em qualquer outro recurso didático Qualidade que não é detalhe ou luxo e sim necessidade e, como tal, deve ser buscada. 
Já a dissertação “A aprendizagem baseada em problemas e o software GeoGebra no ensino das funções matemáticas" de [Silva 2015], a pesquisa foi realizada com alunos do primeiro ano do Ensino Médio de uma escola da rede pública estadual, localizada na zona leste de São Paulo. A proposta para a pesquisa foi trabalhar com uma turma utilizando o software GeoGebra e a abordagem da Aprendizagem Baseada em Problemas, acreditando que o uso de software como ferramenta potencializa a aprendizagem de Matemática.

Esta pesquisa foi importante, para o aprimoramento profissional do professor e pesquisador em sua prática docente, fruto da sua inquietação em se apropriar das potencialidades do uso instrucional das TIC's. Para tanto, mergulhou na integração do software GeoGebra para o ensino de funções matemáticas, como já prescrito pelo currículo oficial do estado de São Paulo; utilizou ABP e o método da pesquisa - ação num processo de intensa reflexão, e provou a maior das mudanças, a própria ressignificação de sua prática docente.

\section{Resultados e Discussões}

De acordo com os objetivos traçados para essa pesquisa, podemos destacar que diante dos artigos, monografias e dissertações foi possível mapear diversos softwares utilizados para o ensino de matemática e como esses programas facilitam o processo de ensino aprendizagem dentro da sala de aula, despertando o interesse e a atenção dos alunos.

No decorrer da pesquisa e descrição dos softwares, entendemos como cada um pode ser utilizado nas diversas ramificações da matemática, e com isso, podem ser trabalhados em todas as series do ensino fundamental e médio.

Os principais softwares encontrados foram: Régua e Compasso, SuperLogo, Winplot, Winmat, Ms. Lindquist, Venn, NCX, Calques 3D, Wingeom, GeoGebra, Resolução de Sistemas Lineares, OpenOficce.org Calc. e Círculo Trigonométrico,

O GeoGebra foi o principal software citado entre as pesquisas encontradas, por ser gratuito, de fácil acesso, podendo ser aplicado nos processos de ensino e de aprendizagem nas disciplinas de Geometria, Álgebra e Cálculo. Este software permite a criação de figures em 2D e 3D. O GeoGebra é um sistema de geometria dinâmica que permite realizar construções com pontos, vetores, segmentos, retas, seções cônicas e funções que podem ser modificadas dinamicamente [Souza 2010].

\section{Considerações Finais}

A revisão sistemática de literatura teve como objetivo selecionar trabalhos que fizessem o uso de softwares de matemática para o ensino fundamental e médio, com propósitos educacionais, de aprendizagem, buscando identificar a forma com que esses softwares podem auxiliar no ensino e despertar o interesse por parte dos alunos e ajudar o professor a trazer aulas dinâmicas e criativas.

Pode-se notar que os softwares e jogos eletrônicos fazem parte do cotidiano dos jovens e crianças atualmente, por isso, podemos utilizar as novas tecnologias de informação e comunicação como proposta metodológica para o ensino de matemática.

Ao utilizá-los pode-se proporcionar uma interatividade entre o aluno e a tecnologia, e assim, este pode aprender de forma livre e motivadora. Além de adquirir habilidades formadoras constituintes, como pensamento lógico e a construção de estratégias. Particularmente na área da matemática, o uso dos softwares provoca no aluno a participação e a motivação para compreender os conteúdos. 
A tecnologia é uma das soluções favoráveis para o ensino e aprendizagem, pois quanto mais possibilidades de ambas forem trazidas para o espaço escolar, mais atenção e participação dos "nativos digitais" serão alcançadas.

\section{Referências}

Abreu, A. C. D. (2011). O uso de softwares na aprendizagem da matemática. Cuiabá: Universidade Federal de Mato Grosso.

Batista, S. C. F. (2004). O uso de softwares na aprendizagem da matemática. Trabalho de Conclusão de Curso (dissertação). Universidade Estadual do Norte Fluminense UENF, Rio de Janeiro.

Brasil. (1997) Secretaria de Educação Fundamental (SEF) Parâmetros Curriculares Nacionais: matemática. Brasília, DF: MEC/SEF. Disponível em: http://portal.mec.gov.br/seb/arquivos/pdf/livro01.pdf. Acesso em maio de 2019.

Brasil. (1988). Constituição da República Federativa do Brasil [recurso eletrônico]. Brasília: Supremo Tribunal Federal, Secretaria de Documentação, 2017. 514p. Atualizada até a EC n. 96/2017.

Contri, R. F. F., Retzlaff, E. and Klee, L. A. (2011) Uso de softwares matemáticos como facilitador da aprendizagem. Rio Grande do Sul.

Cordeiro, A. M., de Oliveira, G. M., Rentería-TCBC-RJ, J. M., \& Guimarães-TCBC-RJ, C. A. (2007). Revisão sistemática: uma revisão narrativa.

Costa, F. J. C., Costa, A. J. C., Rodrigues, A. P. and Vasconcellos, T. F. (2016) O uso de softwares aplicativos no ensino da Matemática: a tecnologia como figura de mediação pedagógica. Natal.

FANTI, E. L. C. (2010). Utilizando o software Geogebra no ensino de certos conteúdos matemáticos. Bienal da Sociedade Brasileira de Matemática, 5, 1-18.

Machado, N. J. (1994). Epistemologia e didática: as concepções de conhecimento e inteligência e a prática docente. Cortez, São Paulo.

MORAIS, R. X. T. (2003). Software educacional: a importância de sua avaliação e do seu uso nas salas de aula. Monografia, Faculdade Lourenço Filho.

Nogueira, T. C. A., Cardoso, M. C. S. A., Figueira-Sampaio, A. S., Santos, E. E. F., \& Carrijo, G. A. (2013). Software educativos gratuitos para o ensino de Matemática. In Anais do Congresso Internacional de Informatica Educativa-TISE 2013.

Pacheco, J. A. D., \& BARROS, J. V. (2013). O uso de softwares educativos no ensino de matemática. Revista de Estudos Culturais e da Contemporaneidade, Garanhus, (8), 513.

Pereira, L. R. (2014) O Ensino e a Aprendizagem da Matemática mediada por softwares Educativo na forma de Objetos de Aprendizagem. Paraná.

SALVAT, Begoña Gros. (1998) Direção Ana Teberosky e Liliana Tolchinsky; tradução: Francisco Franke Settineri. Substratum: Temas Fundamentais em Psicologia e Educação. v. 2, n. 5. Artmed, Porto Alegre.

SILVA, João Paulo Martins da. RIBEIRO, Aretha Cristina. ARAÚJO, Tacildo de Souza. (2018) O uso de softwares no ensino da matemática: entre o modismo e o uso inteligente. Olinda. 
Silva, M. F., Costa Cortez, R. D. C., \& de Oliveira, V. B. (2013). Software Educativo como auxílio na aprendizagem da matemática: uma experiência utilizando as quatro operações com alunos do $4^{\circ}$ Ano do Ensino Fundamental I. Educação, Cultura e Comunicação, 4(7).

Silva, J. C. E. A aprendizagem baseada em problemas e o software Geogebra no ensino das funções matemáticas. 2015. 29 f. Mestrado Profissional em Ensino de Ciências e Matemática. (Dissertação) - Universidade Cruzeiro do Sul. 2015.

Tederke, A. R. Fortes, P. R. and Silveira, S. R. Um Estudo de Caso Envolvendo a Aplicação de um Software Educacional de Geometria Espacial. Mato Grosso do Sul.2016. 